\title{
Role of mesoscopic morphology in charge transport of doped polyaniline
}

\author{
A K MUKHERJEE and REGHU MENON \\ Department of Physics, Indian Institute of Science, Bangalore 560 012, India
}

\begin{abstract}
In doped polyaniline (PANI), the charge transport properties are determined by mesoscopic morphology, which in turn is controlled by the molecular recognition interactions among polymer chain, dopant and solvent. Molecular recognition plays a significant role in chain conformation and charge delocalization. The resistivity of PANI doped by camphor sulfonic acid (CSA)/2acrylo-amido-1-propane sulfonic acid (AMPSA)/dodecyl benzene sulfonic acid (DBSA) is around $0.02 \Omega \mathrm{cm}$. PANI-CSA and PANI-AMPSA show a metallic positive temperature coefficient of resistivity above $150 \mathrm{~K}$, with a finite value of conductivity at $1.4 \mathrm{~K}$; whereas, PANI-DBSA shows hopping transport at low temperatures. The magnetoresistance is positive (negative) for PANI-CSA (PANIAMPSA); and PANI-DBSA has a large positive MR. The behavior of MR suggests subtle variations in mesoscopic morphology between PANI-CSA and PANI-AMPSA.
\end{abstract}

Keywords. Resistivity; magnetoresistance; molecular recognition; morphology.

PACS Nos 72.15.Gd; 72.80.Le; 73.23.-b

\section{Introduction}

In the last decade the semiconducting and metallic properties of organic $\pi$-electron systems have drawn considerable attention both from fundamental and applications point of view [1]. Among various types of $\pi$-electron systems, the electrical and optical properties of conjugated polymers have shown wide range of exciting features, leading towards applications like light emitting diodes, photodiodes, electronic circuits, lasers, sensors, solar cells, etc. After the discovery of metallic polyacetylene in 1977 [2], various $\pi$-conjugated systems like polypyrrole, polythiophene, polyaniline, poly(para)phenylene-vinylene, etc., have been prepared, and its physical properties have been investigated [1]. Since polymeric materials are inherently partially crystalline and partially amorphous, the structure and morphology play significant roles in the electronic properties. Moreover, the size of ordered/disordered regions in conjugated macromolecular $\left[(-\mathrm{C}=\mathrm{C}-\mathrm{C}=\mathrm{C}-\mathrm{C}=\mathrm{C}-)_{n}\right]$ systems can vary from nanoscopic to macroscopic levels, which to a large extent is dependent upon the sample preparation and processing conditions. This complex relationship between structure/morphology and electronic properties has made the understanding of charge transport properties in conducting polymers quite difficult with respect to conventional systems. Moreover, the disorder-induced localization plays a significant role in the metal-insulator (M-I) transition in doped conducting polymers. This scenario is quite well demonstrated in the case of the charge transport properties in doped polyaniline (PANI). 
(a)

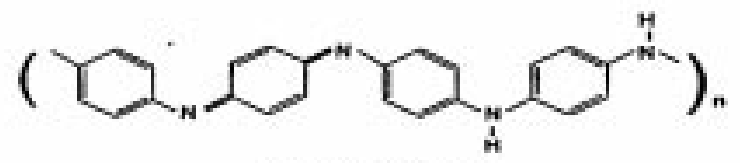

Emeraldine base

(b)

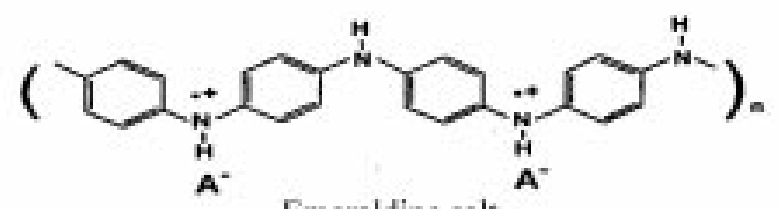

Emeraldine salt

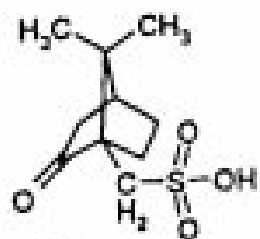

(c)

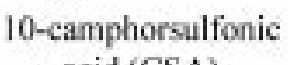

acid (CSA)

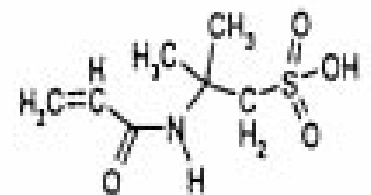

(d) 2-acryloamido-1-propane sulfonic acid (AMPSA)

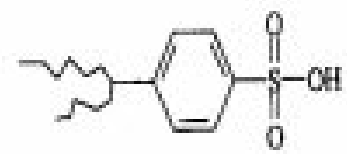

(e)

dodecyl benzene sulfonic acid (DBSA)

Figure 1. Chemical structures of polyaniline and dopants.

The physical properties of PANI have been investigated in the last decade [3]. PANI is a semi-rigid intractable polymer that can be easily obtained by chemical polymerization of aniline. The insulating form of PANI is called emeraldine base, as shown in figure 1a; and upon doping by protonation with acids $\left(\mathrm{H}^{+} \mathrm{A}^{-}\right)$, it is possible to obtain the conductive form of PANI called emeraldine salt, as in figure $1 \mathrm{~b}$.

PANI is a semiconductor with a band gap around $2.6 \mathrm{eV}$, and its band structure is shown in figure 2. The mid-gap polaronic states make it rather different from conventional inorganic semiconductors. Upon doping, the mid-gap level broadens and merges with conduction/valance bands, and this induces the transition from insulator to metal [4,5].

Undoped PANI is sparingly soluble in solvents like $N$-methyl pyrrolidone, and in the doped state the charged chains become quite stiff. As a result it is rather insoluble in common solvents [6]. However, Cao et al [7] made PANI soluble by using a dopant having a surfactant counterion, and this route provided a conductive solution of PANI. This surfactant counterion induced processibility of PANI has helped to obtain high quality free standing films of PANI. Moreover, this has increased the conductivity of doped PANI from $10 \mathrm{~S} / \mathrm{cm}$ to $10^{3} \mathrm{~S} / \mathrm{cm}[7,8]$, and for the first time the intrinsic metallic properties of PANI could be observed $[4,5]$. At the same time, it was realized that various combinations of surfactant counterions and solvents could significantly alter the polymer chain conformation via the molecular recognition interactions, and this can drastically modify the electronic properties of PANI [9]. Moroever, the improved solution route processibility of PANI 


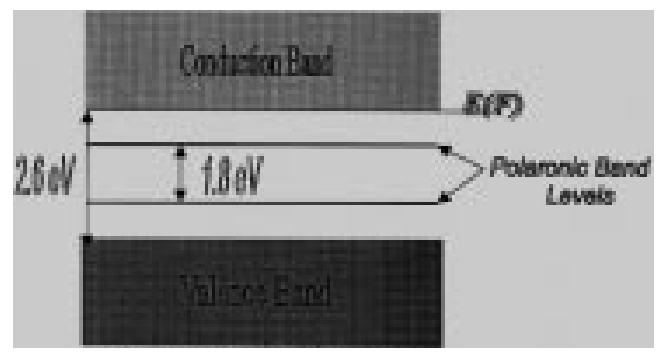

Figure 2. Band diagram of polyaniline.

has enabled to fabricate it in thin films, free standing films, fibres, etc., for various applications in electronic devices, electromagnetic shielding, anti-corrosive coating, artificial muscles [1], etc.

\section{Molecular recognition and mesoscopic morphology}

It is well-known that macromolecular systems are quite complex, since the polymer chains run in all possible directions, get coiled-up, converge-in and diverge-out at various regions. Nevertheless, the charged polyconjugated chains are relatively stiff and the $\pi-\pi$ interactions can easily give rise to fairly large ordered domains in the system. Hence, with respect to conventional polymeric systems, it is possible to have substantial crystalline order in polyconjugated systems. However, the extended or coiled conformation of polymer chains depends to a large extent on the structure and processing of the polymer.

In solution processed PANI, the interactions among polymer chains, surfactant counterion and solvent determine the chain conformation and morphology of the films. Usually the charged polymer chains are polar in nature, and the polarity of counterion and solvent play crucial roles in the chain conformation. Moreover, the steric and functional group interactions contribute to the conformation of chains. In the case of less polar solvents the PANI chain tends to coil up; whereas, in the presence of highly polar solvents the polymer chain forms extended conformation $[9,10]$. Also, polar counterion enhances the extended chain conformation. For e.g., in the presence of a combination of camphor sulfonic acid (CSA, see figure 1c) or 2-acrylo-amido-1-propane sulfonic acid (AMPSA, see figure 1d) in meta-cresol or dichloroacetic acid, the PANI chains tend to form the extended chain conformation [7-10] whereas, the combination of dodecyl benzene sulfonic acid (DBSA, see figure 1e) and xylene or chloroform makes the PANI chains coiled up. This has been confirmed from previous structural studies [11].

This type of molecular level selective interaction is usually called molecular recognition [12]. In a typical molecular recognition process, the interacting entity has a certain amount of molecular information, and the degree of interaction between the entities is determined by the degree of exchange of molecular information that gives rise to the formation of a supramolecular assembly. The phenomenon of molecular recognition is well-known in several biological systems, as a typical case in which a molecular complex is linked by sterically fitting hydrogen bonds (for example, when uracil and 2,6-diacylamino-pyridine 
[13] is modified by nonmesogenic alkyl groups) to form a self-organized supramolecular assembly. Similarly, it is known that in polyalkylthiophenes, the alkyl side-group interactions induce an ordering of the polymer chain backbone [14].

This type of molecular recognition phenomena determines the morphology of films in surfactant counterion processed PANI. The coiled chain conformation facilitates chain entanglements, and the entanglements of a large number of chains give rise to mesoscopic size granular morphology. As a result the films tend to be more amorphous in nature whereas the extended chain conformation is more favorable for the growth of well-ordered mesoscopic size domains, and this increases the volume fraction of crystalline regions [15].

In disordered quasi-one-dimensional systems like conducting polymers the charge transport is mainly determined by the morphology of the system [16]. The bulk charge transport involves both intra- and inter-chain contributions. The defects in polymer chain and weak interchain transfer integral can easily lead to localization of charge carriers with respect to that in the three-dimensional electronic systems. In amorphous films a large number of electronic states are localized, and the interchain transport is mainly via the thermallyassisted hopping of carriers from chain to chain [17] whereas, in films having extended chain morphology, the delocalized electronic states favor coherent interchain diffusion of carriers. As a typical example, the uncoiling and extension of coiled PANI chains by exposing it to polar solvent vapors could transform some of the localized polaronic carriers to free carriers [10]. This demonstrates that subtle level molecular recognition interactions control the mesoscopic scale morphology and that in turn determines the charge transport properties in conducting polymers.

Free standing films of doped PANI samples (thickness $\sim 30$ microns) are prepared by solution casting, drying and peeling off from silicon-wafer substrates. PANI-CSA and PANI-DBSA are prepared from meta-cresol, and PANI-AMPSA is from dichloroacetic acid solution. Four wires are attached to the samples by carbon paint for transport property measurements.

\section{Resistivity and magnetoresistance in doped PANI}

A comparison of the temperature dependence of resistivity and magnetoresistance (MR) of PANI doped by CSA, AMPSA and DBSA has been made in this paper. The room temperature resistivity values of all the three doped PANI samples are around $0.02 \Omega \mathrm{cm}$. The resistivity ratios $\left(\rho_{\mathrm{r}} \sim \rho(1.4 \mathrm{~K}) / \rho(300 \mathrm{~K})\right)$ of PANI-CSA, PANI-AMPSA and PANIDBSA are 3, 2.1 and 173, respectively. The temperature dependence of resistivity of doped PANI samples is shown in figure 3. Both PANI-CSA and PANI-AMPSA show metallic positive temperature coefficient of resistivity (TCR) down to 140 and $120 \mathrm{~K}$, respectively.

Although the room temperature resistivities of all the three doped PANI samples are nearly identical, their temperature dependence is rather different. Firstly, the positive TCR in disordered PANI-CSA and PANI-AMPSA samples is quite surprising, since these systems are at the Mott minimum metallic conductivity $(\sim 50 \mathrm{~S} / \mathrm{cm})[16,17]$, whereas, PANIDBSA shows nearly temperature independent resistivity down to $10 \mathrm{~K}$, and then it abruptly increases at lower temperatures. Secondly, PANI-CSA and PANI-DBSA have a finite conductivity $(\sim 25 \mathrm{~S} / \mathrm{cm})$ at $1.4 \mathrm{~K}$, and PANI-DBSA goes to the insulating state below $10 \mathrm{~K}$.

The data analysis indicates that PANI-CSA and PANI-DBSA are at the critical regime of disorder-induced M-I transition, and PANI-DBSA shows the typical Mott's variable range 


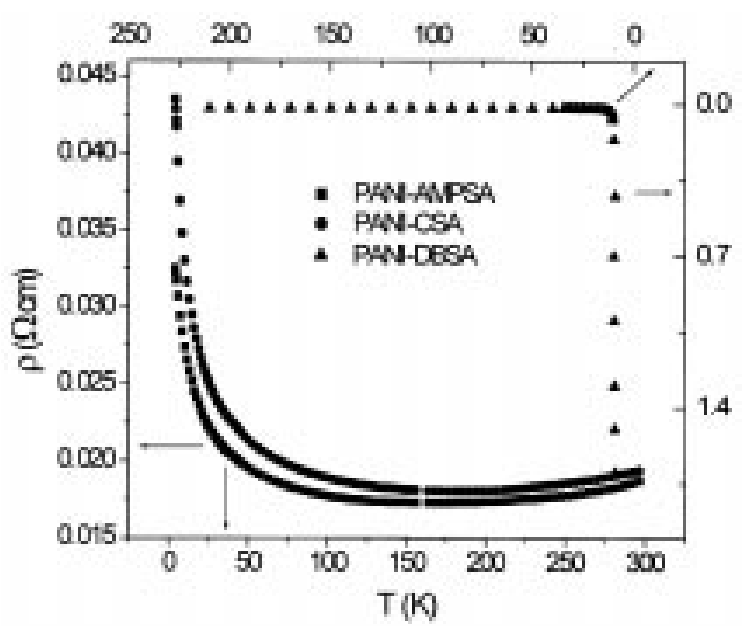

Figure 3. Resistivity vs temperature of doped polyaniline.

hopping transport [18]. This suggests that subtle variations in the size and volume fraction of ordered mesoscopic domains can drastically affect the charge transport through the delocalized electronic states, since it is known that the mean free path in disordered quasi-one-dimensional systems is quite sensitive to the interchain transfer integral [19]. In this context, the important role played by molecular recognition in controlling the mesoscopic scale morphology is quite evident, and that in turn determines the charge transport properties in conducting polymers.

The transverse magnetoresistance (MR) of doped PANI samples at $1.4 \mathrm{~K}$ is shown in figure 4. Although the room temperature resistivity and TCR of both PANI-CSA and PANI-AMPSA are nearly identical, the behavior of MR is quite different. In PANI-CSA, a positive MR (nearly $18 \%$ at $1.4 \mathrm{~K}$ and $6 \mathrm{~T}$ ) was observed, whereas in PANI-AMPSA, the MR is negative (nearly $4 \%$ at $1.4 \mathrm{~K}$ and $6 \mathrm{~T}$ ), as shown in figure 4 . The positive MR in PANI-CSA is rather similar to that observed in highly disordered systems near the M-I transition [20]. Although the extent of disorder is expected to be similar in PANI-AMPSA to that in PANI-CSA (inferred from the TCR), the negative MR is quite identical to that observed in less disordered metallic systems due to the weak localization correction to conductivity [20,21]. Since, MR probes the local charge carrier dynamics, it gives insight into the microscopic transport property parameters (e.g. scattering lengths, relaxation time, etc.). In order to observe the negative MR in PANI-AMPSA, the charge delocalization in mesoscopic metallic domains of PANI-AMPSA should be better with respect to that in PANI-CSA. Hence, the MR data indicate an important role of AMPSA counterion in ordering the PANI chains via the molecular recognition process, and that leads to the delocalization of charge carriers. Although the temperature dependence of resistivity could not make out this difference between PANI-CSA and PANI-AMPSA, the MR data was quite useful in identifying the subtle morphological variations among them. The large positive MR (nearly $150 \%$ at $1.4 \mathrm{~K}$ and $6 \mathrm{~T}$ ) in PANI-DBSA is typical to that expected in case 


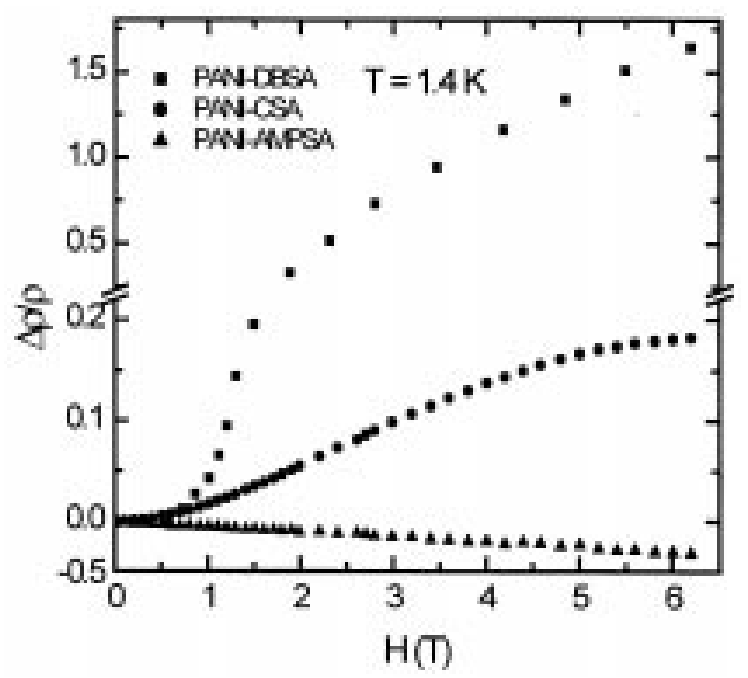

Figure 4. Magnetoresistance of doped polyaniline.

of variable range hopping transport, since the overlap of wave functions shrinks in the presence of a magnetic field and as a result the hopping of carriers from one localized state to another decreases [17].

\section{Summary}

The preliminary results on the role of molecular recognition in controlling the mesoscopic scale morphology and charge transport properties in doped PANI suggest that the electronic properties in complex systems like conducting polymers is quite sensitive to the structural/morphological features. Though the room temperature resistivity of all the three doped PANI samples are nearly identical, their TCR and MR are quite different. PANICSA and PANI-AMPSA are just on the metallic side of the M-I transition, and PANIDBSA is on the insulating side. The sign of MR is positive and negative in PANI-CSA and PANI-AMPSA, respectively. This indicates that the ordered mesoscopic regions in PANI-AMPSA are more metallic with respect to that in PANI-CSA. Nevertheless, further studies are essential to fully understand the intriguing correlation between structural/morphological features and charge transport properties.

\section{Acknowledgements}

We offer our congratulations to Prof. N Kumar on the occasion of his 60th birthday, and express our appreciation of the great contribution he has made to the field of disordered systems. 


\section{References}

[1] Handbook of conducting polymer edited by T A Skotheim, R L Elsenbaumer and J R Reynolds, 2nd ed. (Marcel Dekker, NY, 1998)

[2] C K Chian, C R Fincher Jr, Y W Park, A J Heeger, H Shirakawa, E J Louis, S C Gau and A G MacDiarmid, Phys. Rev. Lett. 39, 1098 (1977)

[3] F Wudl, R O Angus Jr, F L Lu, P M. Allemand, D J Vachon, M Nowak, Z X Liu and A J Heeger, J. Am. Chem. Soc. 109, 3677 (1987)

[4] M Reghu, Y Cao, D Moses and A J Heeger, Phys. Rev. B47, 1758 (1993)

[5] M Reghu, C O Yoon, D Moses, A J Heeger and Y Cao, Phys. Rev. B48, 17685 (1993)

[6] M Angelopoulos, A Ray, A G MacDiarmid and A J Epstein, Synth. Met. 21, 21 (1987)

[7] Y Cao, P Smith and A J Heeger, Synth. Met. 48, 91 (1992)

[8] P N Adams, P Devasagayam, S J Pomfret, L Abell and A P Monkman, J. Phys. Condens. Matter 10, 8293 (1998)

[9] O T Ikkala, L-O Pietila, L Ahjopalo, H Osterholm and P J Passiniemi, J. Chem. Phys. 103, 9855 (1995)

[10] Y Xia, A G MacDiarmid and A J Epstein, Macromolecules 27, 7212 (1994)

[11] C Y Yang, Y Cao, P Smith and A J Heeger, Synth. Met. 53, 293 (1993)

[12] T Vikki, L-O Pietila, H Osterholm, L Ahjopalo, A Takala, A Toivo, K Levon, P Passiniemi and O T Ikkala, Macromolecules 29, 2945 (1996)

[13] M J Brienne, J Gobard, J M Lehn and I Stibor, J. Chem. Soc. Chem. Commun. 24, 1864 (1989)

[14] R D McCollough, R D Lowe, M Jayaraman and D L Anderson, J. Org. Chem. 58, 904 (1993)

[15] A G MacDiarmid and A J Epstein, Synth. Met. 65, 103 (1994)

[16] Reghu Menon, Co Yoon, D Moses and A J Heeger in Handbook of conducting polymer edited by T A Skotheim, R L Elsenbaumer and J R Reynolds, 2nd ed. (Marcel Dekker, NY, 1998)

[17] N F Mott, Metal insulator transition, 2nd ed. (Taylor and Francis, London, 1990)

[18] A K Mukherjee and Reghu Menon (to be published)

[19] S Stafström, Phys. Rev. B51, 4137 (1995)

[20] A L Efros and M Pollak, Electron-electron Interactions in disordered systems (North Holland, Amsterdam, 1985)

[21] P A Lee and T V Ramakrishnan, Rev. Mod. Phys. 57, 287 (1985) 